Accretion Phenomena and Related Outflows, IAU Colloquium 163

ASP Conference Series, Vol. 121, 1997

D.T. Wickramasinghe, L. Ferrario, and G.V. Bicknell, eds.

\title{
Radiation Hydrodynamics and Soft X-Ray Emission of Accreting Magnetic White Dwarfs
}

\author{
K. Beuermann, A. Fischer, Th. Rousseau \\ Universitäts-Sternwarte, Geismarlandstraße 11, Göttingen, Germany
}

\begin{abstract}
Observed cyclotron spectra of AM Herculis binaries imply a large range of mass flow rates in the accretion spot on the white dwarf. We derive this $\dot{m}$-distribution for the case of UZ For. For AM Her stars with different field strength $\mathrm{B}$, such a distribution is shifted in $\dot{m}$ proportional to $B$. This fact can account for the observed dominance of bremsstrahlung at low $B$ and of quasi-blackbody emission at high $B$ values.
\end{abstract}

In accreting magnetic white dwarfs, the atmosphere is heated by a stream of free falling protons with mass flow rate $\dot{m}$. For $\dot{m} \lesssim 10^{-1} \mathrm{~g} \mathrm{~cm}^{-2} \mathrm{~s}^{-1}$, the protons loose their energy by Coulomb encounters with atmospheric electrons (bombardment solution) whereas for higher $\dot{m}$ a radiative shock forms (Woelk and Beuermann 1992, 1996). The principal result of their radiation-hydrodynamic calculations is the strong dependence of the peak electron temperature $T_{\max }$, the column density $x_{s}$ of the post-shock flow, and the fractional energy radiated as bremsstrahlung or cyclotron radiation on both $B$ and $\dot{m}$. Calculating cyclotron spectra with the derived temperature distributions, we found that different mass flow rates contribute quite differently to individual cyclotron harmonics (Rousseau et al. 1996). The presence of several cyclotron harmonics in an observed spectrum requires, therefore, a range of mass flow rates (typically $10^{-4} \leq \dot{m} \leq 10^{-1} \mathrm{~g} \mathrm{~cm}^{-2} \mathrm{~s}^{-1}$ for the optical range and up to $10 \mathrm{~g} \mathrm{~cm}^{-2} \mathrm{~s}^{-1}$ for the UV range).

As an example, we have applied the theory to the low and high state spectra of UZ For (Fig. 1). The UV flux shown in the upper panel was derived from Stockman and Schmidt (1996) and represents an estimate of the general level of the cyclotron component in the UV. Table 1 gives the contributions $\Delta A$ and $\Delta \dot{M}$ to the area of the spot and to the accretion rate $\dot{M}$ per unit in $\log \dot{m}$ required to fit the spectra. The high-state value of $\dot{M}\left(\sim 10^{14} \mathrm{~g} \mathrm{~s}^{-1}\right)$ falls far below the soft-X-ray-derived value of $\sim 2 \times 10^{15} \mathrm{~g} \mathrm{~s}^{-1}$ (Beuermann and Burwitz 1995). Consequently, more than $90 \%$ of $\dot{M}$ must be due to flow rates $\dot{m} \gtrsim 10 \mathrm{~g} \mathrm{~cm}^{-2} \mathrm{~s}^{-1}$. This is the regime where "blobby accretion" takes over, the shock becomes submerged in the photosphere (Beuermann and Woelk 1996) and the energy emerges almost exclusively as soft X-rays. This is confirmed by the fact that UZ For is completely dominated by soft X-ray emission (Beuermann and Burwitz 1995).

A simple picture may be employed to understand why high-field systems as UZ For are copious producers of soft X-rays and lack a strong bremsstrahlung component (the so called "soft X-ray puzzle"). For AM Her stars with different field strengths $B$, the $\dot{m}$ distribution is centered at mean values which increase 
proportional to $B$ (Beuermann and Woelk 1996). For increasing $B$, therefore, a larger fraction of the $\dot{m}$ distribution falls into the regime of blobby accretion. At the same time, for the remaining fraction Woelk and Beuermann (1996) demonstrated that bremsstrahlung is increasingly supressed compared with cyclotron radiation. The result is a continous drop of $F_{\text {brems }} / F_{\text {soft-x }}$ following closely the observed relative flux variation (e.g. Beuermann and Burwitz 1995).

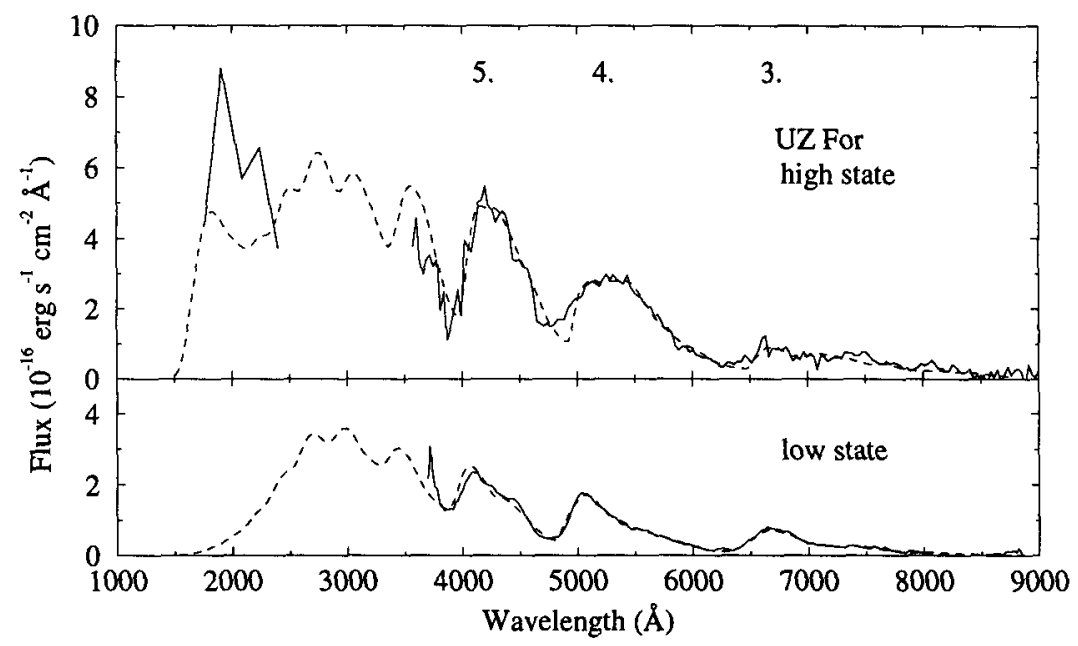

Figure 1. Observed (solid) and calculated (dashed) cyclotron spectra of UZ For in the high state (top) and the low state (bottom).

Table 1. Accreting areas $\Delta A\left(10^{15} \mathrm{~cm}^{2}\right)$ and rates $\Delta \dot{M}\left(10^{13} \mathrm{~g} \mathrm{~s}^{-1}\right)$.

\begin{tabular}{lccll}
\hline & \multicolumn{2}{c}{ low state } & \multicolumn{2}{c}{ high state } \\
$\dot{m}$ & $\Delta A$ & $\Delta \dot{M}$ & $\Delta A$ & $\Delta \dot{M}$ \\
\hline 3.2 & $\sim 0$ & $\sim 0$ & 0.01 & 3 \\
0.32 & 0.05 & 1.6 & 0.08 & 2.6 \\
0.032 & 0.26 & 0.83 & 0.74 & 2.4 \\
0.0032 & 1.14 & 0.36 & 2.4 & 0.77 \\
0.00032 & 3.4 & 0.11 & 1.8 & 0.06 \\
Sum & 4.85 & 2.90 & 5.03 & 8.83 \\
\hline
\end{tabular}

\section{References}

Beuermann, K., Burwitz, V., 1995, ASP Conf. Ser., Vol. 85, 99

Beuermann, K., Woelk, U., 1996, IAU Coll. 158, in press

Rousseau, Th., Fischer, A., Beuermann, K., Woelk, U., 1996, A\&A, 310, 526

Stockman, H.S., Schmidt, G.D., 1996, ApJ, in press

Woelk, U., Beuermann, K., 1992, A\&A, 256, 498

Woelk, U., Beuermann, K., 1996, A\&A, 306, 232 